\title{
Cannabinoid type 1 receptor mediates depot-specific effects on differentiation, inflammation and oxidative metabolism in inguinal and epididymal white adipocytes
}

\author{
IV Wagner, N Perwitz, M Drenckhan, H Lehnert and J Klein \\ Department of Internal Medicine I, University of Luebeck, Luebeck, Germany
}

\begin{abstract}
Objective: The endocannabinoid system is a major component in the control of energy metabolism. Cannabinoid 1 (CB1)receptor blockade induces weight loss and reduces the risk to develop the metabolic syndrome with its associated cardiovascular complications. These effects are mediated by central and peripheral pathways. Interestingly, weight loss is mainly achieved by a reduction of visceral fat mass. We analyzed fat depot-specific differences on adipocyte differentiation, inflammation and oxidative metabolism in CB1-receptor knockout cells.

Materials and methods: We used newly generated epididymal/inguinal adipose cell lines from CB1-receptor knockout mice. Differences in differentiation were measured by fat-specific Oil Red $\mathrm{O}$ staining and quantitative analysis of key differentiation markers. Induction of apoptosis was evaluated by cell death detection and investigation of p53 phosphorylation. Inflammation markers were quantified by real-time PCR. For analyzing the process of transdifferentiation we measured oxygen consumption and mitochondrial biogenesis.

Results: Differentiation was reduced in visceral adipocytes from CB1-receptor knockout mice as compared with wild-type controls. Moreover, we found an induction of apoptosis in these cells. In contrast, subcutaneous adipocytes from CB1-receptor knockout mice showed an accelerated differentiation and a reduced rate of apoptosis. Inflammation was increased in visceral fat cells, as analyzed by the expression pattern of interleukin-6, monocyte chemoattractant protein 1 (MCP-1), tumor necrosis factor- $\alpha$, whereas in subcutaneous adipocytes these markers were decreased. Furthermore, subcutaneous CB1-receptor knockout cells were more sensitive toward a conversion into a brown fat phenotype. Uncoupling protein-1 as well as PGC-1 $\alpha$ expression was significantly elevated. This was accompanied by an increase in mitochondrial biogenesis and oxygen consumption.

Conclusion: In conclusion, we found depot-specific effects on differentiation, apoptosis, inflammation and oxidative metabolism in CB1-receptor knockout cells. Thus, CB1-receptor-mediated pathways differentially target adipose tissue depots to a dual effect that minimizes cardiometabolic risk, on the one hand, by diminishing visceral fat, and that enhances thermogenesis in subcutaneous adipocytes, on the other.
\end{abstract}

Nutrition and Diabetes (2011) 1, e16; doi:10.1038/nutd.2011.12; published online 5 September 2011

Keywords: CB1-receptor knockout mice; depot-specific effects in adipose tissue; adipogenesis; apoptosis; inflammation; transdifferentiation

\section{Introduction}

Obesity and related cardiovascular complications are one of the major health problems at present. Obesity is commonly seen as a disorder of energy balance, where energy

Correspondence: Professor J Klein, Department of Internal Medicine I, University of Luebeck, Ratzeburger Allee 160, 23538 Luebeck, Germany. E-mail: j.klein@uni-luebeck.de

Received 2 March 2011; revised 14 June 2011; accepted 10 July 2011 intake exceeds energy expenditure. ${ }^{1,2}$ Alteration of adipocyte function is a critical component in the pathogenesis of obesity and its related cardiometabolic complications. ${ }^{3}$

The endocannabinoid (EC) system is emerging as a key player in the control of energy homeostasis. ${ }^{4}$ It stimulates a positive energy balance via both central and peripheral pathways. The cannabinoid 1 (CB1) receptor is distributed in brain areas and is associated with motor control, emotional responses, motivational behavior and energy homeostasis. In the periphery, the same receptor is expressed in adipose 
tissue, pancreas, liver-gastrointestinal tract, skeletal muscles, the heart and in the reproduction system. Stimulation of the CB1-receptor with a specific agonist WIN 55212.2 has a negative impact on thermogenesis in brown adipocytes and favors a positive energy balance by regulating glucostatic adipokines. ${ }^{5}$ In contrast, CB1-receptor-deficient mice have less fat mass than their wild-type littermates and are protected against diet-induced obesity. ${ }^{6}$ Blocking the receptor with a selective CB1-receptor antagonist rimonabant induces weight loss, increases insulin sensitivity, and improves cardiovascular risk factors in overweight patients. ${ }^{7}$ The mechanisms responsible for these effects remain only partially elucidated. Recently, Tedesco et al. ${ }^{8}$ and our group presented several lines of evidence, that a direct blockade of peripheral CB1-receptor action in adipose tissue induces transdifferentiation in white adipocytes toward a thermogenic active brown fat cell phenotype and enhances insulin sensitivity. ${ }^{3}$

Different depots of white adipose tissue appear to exert different endocrine functions. ${ }^{9-11}$ Excess of visceral abdominal fat is known to be metabolically harmful and is associated with high levels of cholesterols, hyperlipidemia, insulin resistance and a high risk to develop atherosclerotic cardiovascular disease. ${ }^{9-11}$ By contrast, subcutaneous fat may behave differently and even have a protective role. ${ }^{12-15}$ Porter et al. ${ }^{10}$ concluded after having analyzed more than 3001 patients (Framingham heart study) that although adiposity increases the absolute risk of metabolic and cardiovascular disease, abdominal subcutaneous fat is not associated with a linear increase in the prevalence of factors that are related to obesity. Indeed, in the case of high triglycerides, subcutaneous adipose tissue may actually be a protective fat depot in obese humans. ${ }^{10,13}$ Visceral adipose tissue and subcutaneous adipose tissue differ not only in their anatomic location and metabolic function but also in their cytokine secretion profile. Subcutaneous adipose tissue releases two to three times more leptin than visceral adipose tissue; ${ }^{16}$ whereas visceral adipose tissue secretes more adiponectin, interleukin (IL)-6 and -8, plasminogen activator-inhibitor 1 , and angiotensin than does subcutaneous adipose tissue. ${ }^{17}$

Finally brown adipose tissue has a role in adaptive thermogenesis, the part of energy expenditure induced by cold exposure or diet. ${ }^{18-21}$ Brown adipose tissue dissipates energy by uncoupling oxidative phosphorylation from ATP production. This process is mediated by uncoupling protein-1 (UCP-1). UCP-1 expression, in turn, is critically regulated by the transcriptional coactivator PGC- $1 \alpha$, which increases the transcriptional activity of peroxisome proliferatoractivated receptor $\gamma$ (PPAR $\gamma$ ) on the UCP-1 promoter. ${ }^{22}$ Ectopic expression of PGC- $1 \alpha$ in white adipose cells activates expression of UCP-1, key mitochondrial enzymes of the respiratory chain, and also enhances the cellular mitochondrial DNA. ${ }^{23}$ PGC- $1 \alpha$ is expressed at higher levels in brown adipose tissue than in white adipose tissue, and its expression is increased in response to cold exposure and $\beta$-adrenergic stimulation. PPAR $\gamma$ and its coactivators, PGC- $1 \alpha$ and SRC-1, influence brown adipocyte metabolism and development. Tiraby and Langin ${ }^{1}$ showed evidence to support the concept of an alteration in energy balance through a conversion of white to brown adipose tissue. Emergence of brown fat cells in white fat depots is associated with a lean phenotype in transgenic mouse models. ${ }^{24,25}$ Transgenic expression of PRDM 16, at physiological levels, in white fat depots stimulates the formation of brown fat cells. ${ }^{26}$ It seems therefore possible that therapeutic strategies aiming at altering the phenotype of white adipocytes could be developed or used for the treatment of obesity.

The peripheral mechanisms responsible for a reduction of visceral adipose tissue and the induction of a negative energy balance when blocking the CB1-receptor remain only partially elucidated. Here, we demonstrate depotspecific effects in differentiation, apoptosis, inflammation and oxidative metabolism in subcutaneous compared with visceral adipocytes of CB1-receptor knockout mice.

\section{Materials and methods}

\section{Materials}

Antibody against PGC-1 $\alpha$, phospho-p53 (Ser15), p53 and Pref-1 were ordered from Cell Signaling Technology Inc. (Beverly, MA, USA). The UCP-1, cannabinoid type 1 receptor and actin antibodies were purchased from Chemicon International (Temecula, CA, USA). The antibodies used against AP2 and PPAR $\gamma$ were from Santa Cruz Biotechnology Inc. (Santa Cruz, CA, USA). Primers for quantitative real-time PCR were ordered from Biometra (Goettingen, Germany). Glucose uptake assays were performed with 2-deoxy-3H glucose from NEN Life Technologies (Deirich, Germany). All other chemicals were from Sigma-Aldrich Co. (St Louis, MO, USA), unless stated otherwise.

\section{Methods}

Generation of adipose cell lines. Periepididymal and inguinal adipose tissues were taken from CB1-receptor knockout and wild-type mice.

Adipose tissue was taken from periepididymal and inguinal regions. After collagenase digestion (1 mg collagenase in $1 \mathrm{ml}$ isolation buffer containing $0.123 \mathrm{mM} \mathrm{NaCl}$, $5 \mathrm{mM} \mathrm{KCl}, 1.3 \mathrm{mM} \mathrm{CaCl}_{2}, 5 \mathrm{mM}$ glucose, $100 \mathrm{mM}$ 4-(2hydroxyethyl)-1-piperazineethanesulfonic acid, $100 \mathrm{U} \mathrm{ml}^{-1}$ penicillin/streptomycin and $4 \%$ bovine serum albumin), cells were transferred to culture plates and grown to 60-70\% confluence. Cells were immortalized with SV40T antigen. Selection began after $72 \mathrm{~h}$ with puromycin $\left(2 \mu \mathrm{g} \mathrm{ml}^{-1}\right)$ and was maintained for 3 weeks. The study was conducted according to the NIH guidelines for the care and use of laboratory animals, and was authorized by the local regulatory authority (Ministerium fuer Landwirtschaft, Umwelt und laendliche Raeume des Bundeslandes Schleswig-Holstein).

\section{Cell culture}

Immortalized wild-type cells as well as newly generated CB1-receptor knockout cell lines from epididymal and 
inguinal depots were differentiated in Dulbecco's modified Eagle's medium supplemented with $20 \%$ fetal bovine serum, $4.5 \mathrm{gl}^{-1}$ glucose, $20 \mathrm{nM}$ insulin and $1 \mathrm{nM}$ triiodthyronine (differentiation medium) in a humified atmosphere of 5\% $\mathrm{CO}_{2}$ at $37^{\circ} \mathrm{C}$. When preadipocytes reached confluence, cell differentiation was induced by $250 \mu \mathrm{M}$ indomethacine, $500 \mu \mathrm{M}$ isobutylmethhylxanthine and $2 \mu \mathrm{g} \mathrm{ml}^{-1}$ dexamethasone for $24 \mathrm{~h}$ (induction medium). Subsequently, cells were grown in differentiation medium for another 6 days, depending on the experiments performed. ${ }^{27,28}$

\section{Western blot analysis/immunoblotting}

After washing the cells with ice-cold phosphate-buffered saline, proteins were isolated using cell lysis buffer containing $2 \mathrm{mM}$ vanadate, $10 \mu \mathrm{g} \mathrm{ml}^{-1}$ aprotinin, $10 \mu \mathrm{g} \mathrm{ml}^{-1}$ leupeptin and $2 \mathrm{~mm}$ phenylmethylsulphonylfluoride. The protein content was quantified using the Bradford protein assay according to the manufacturer's instructions (Bio-Rad, Munich, Germany). Proteins were separated by SDS-polyacrylamide gel electrophoresis and transferred to nitrocellulose membranes (Schleicher and Schuell, Dassel, Germany). Membranes were blocked with rinsing buffer (10 mM Tris, $150 \mathrm{~mm} \mathrm{NaCl}$ and $0,05 \%$ Tween, $\mathrm{pH}$ 7.2) including $3 \%$ bovine serum albumin or $5 \%$ skim milk at $4{ }^{\circ} \mathrm{C}$ overnight. Protein bands were visualized using chemiluminescence (Perkin-Elmer GMbH, Rodgau-Jügesheim, Germany) and enhanced chemiluminescence films (Amersham Pharmacia Biotech, Freiburg, Germany). The subsequent quantification was performed using Quantity One Software (Bio-Rad). Gel loading was normalized using actin as a control protein.

\section{RNA isolation and quantitative real-time PCR}

Total RNA was isolated using QIAzol reagent (Qiagen, Hilden, Germany). To optimize the RNA quality a clean up and DNase digestion were performed using the NucleoSpin RNA II Kit (Macherey-Nagel, Dueren, Germany). Quality of RNA was tested by photometric analysis and agarose gel electrophoresis. An amount of $2 \mu \mathrm{g}$ total RNA was reverse transcribed using Superscript II (Invitrogen, Karlsruhe, Germany) and an oligo p(DT)15 primer (Roche Molecular Biochemicals, Mannheim, Germany) in the presence of RNase inhibitor (Roche Molecular Biochemicals). Quantitative real-time PCR were performed using $1 \times$ SYBR Premix Ex Taq (TaKaRa Bio Europe, Saint-Germaine-Laye, France) in a Mastercycler ep realplex (Eppendorf $\mathrm{GmbH}$, Hamburg, Germany). PCR for all targets was performed as follows: initial denaturation at $95^{\circ} \mathrm{C}$ for $300 \mathrm{~s}, 40$ cycles with $95^{\circ} \mathrm{C}$ for $20 \mathrm{~s}, 60^{\circ} \mathrm{C}$ for $30 \mathrm{~s}$. Specific amplification was confirmed by producing melting curve profiles and by subjecting the amplification products to agarose gel electrophoresis. 36B4 served as a housekeeping gene. Relative quantification was done by using the Mastercycler ep realplex software based on the CT method $\left(\Delta \Delta C_{t}\right.$ method software for relative quantification). ${ }^{29}$ Primer sequences are available on request.
Oil Red O staining

Cells were cultivated in 10-cm plates and fat-specific staining was performed with Oil Red $O$ at days 0, 3 and 6 after induction. For this purpose, adipocytes were washed twice with phosphate-buffered saline and cells were subsequently fixed with $10 \%$ formalin for at least $20 \mathrm{~min}$. Afterwards, cells were exposed to Oil Red $\mathrm{O}$ for $1 \mathrm{~h}$ at room temperature (stocking solution: $0.5 \mathrm{~g}$ Oil Red $\mathrm{O}$ in $100 \mathrm{ml}$ isopropanol; working solution: $60 \%$ stocking solution plus $40 \% \mathrm{H}_{2} \mathrm{O}$ ). To remove the staining solution, cells were washed several times with distilled water. Representative macroscopic as well as microscopic pictures at a 40-fold magnification were taken using a digital camera (Olympus E330, Olympus Imaging Europe, Hamburg, Germany). For densitometric analysis, Oil Red $\mathrm{O}$ stain was removed by incubating the cells with isopropanolol for $15 \mathrm{~min}$. Optical density was measured at a wavelength of $500 \mathrm{~nm}$ in a photometer.

\section{Glucose uptake}

Fully differentiated cells were serum deprived $24 \mathrm{~h}$ before the experiment. After washing the cells twice with Krebs-Ringer buffer, cells were incubated with or without insulin $(1 \mathrm{nM}, 10 \mathrm{nM}$ or $100 \mathrm{nM})$ for $30 \mathrm{~min}$. Then a mix of $1 \mathrm{~mm}$ 2-desoxyglucose and $50 \mu \mathrm{Ciml}^{-1} 2$-desoxy-3H glucose was added for additional $3 \mathrm{~min}$. Cells were washed in phosphatebuffered saline, and lysed with $0.1 \%$ SDS. The incorporated radioactivity was determined by liquid scintillation counting.

\section{Apoptosis}

A photometric enzyme immunoassay for the qualitative and quantitative in vitro determination of cytoplasmic histoneassociated DNA fragments after cell death was performed using a cell death detection ELISA plus kit (Roche Molecular Biochemicals, Mannheim, Germany) according to the manufactures's instructions.

\section{Oxygen consumption}

The respiration rate of preadipocytes and adipocytes was measured by using a Clark-type oxygen electrode (Oxygraph System, Hansatech, England). Adipocytes at days 0 and 6 of differentiation were detached off the plate and trypsinated. Trypsin was stopped with medium and the suspension was centrifuged for $3 \mathrm{~min}$ (1500 r.p.m.) rinsed with phosphatebuffered saline. The pellet was then resuspended in Dulbecco's modified Eagle's medium without supplements. Each sample was analyzed by incubating at least $10^{6}$ cells over a period of 10 min. Measurement was made in a magnetically stirred chamber, connected to a water circulation system to maintain a stable chamber temperature $\left(37^{\circ} \mathrm{C}\right)$. The incoming signals were detected by software from Hansatech and converted into graphs. The rate of consumption was normalized against the number of living cells, which were stained with Trypan blue and were then counted in a Neubauer's counting chamber. 
Flow cytometry

Adipocytes at day 6 of differentiation were trypsinated, washed at least twice with phosphate-buffered saline and were then fixed in $4 \%$ paraformaldehyde for at least $30 \mathrm{~min}$. Labelling was done using $100 \mathrm{~nm}$ MitoFluor Green (Invitrogen) for $20 \mathrm{~min}$ at room temperature as described by the manufacturer. After staining the fluorescence intensity of the cells was determined by FACS Canto (Becton and Dickinson Biosciences, San Jose, CA, USA) and analyzed by the Flow Software (Tree Star, Ashland, OR, USA).

\section{Statistical analyses}

Statistical analyses were performed with Sigma Plot software (SPSS Science, Chicago, IL, USA). Results are presented as mean values \pm s.e.m. Statistical significance was determined using the unpaired Student's $t$-test. $P$-values $<0.05$ were considered significant, and those $<0.01$ were defined as highly significant.

\section{Results}

Depot-specific effects of CB1-receptor knockout on adipocyte differentiation

In epididymal adipocytes of CB1-receptor knockout, we found a lack of differentiation compared with controls, whereas inguinal CB1-receptor knockout cells demonstrated an accelerated lipid storage compared with inguinal wildtype cells as assessed by microscopic pictures after Oil Red O staining (Figure 1). These observations were confirmed by densitometric analysis of Oil Red $\mathrm{O}$ staining (Figures 2a and b). Lipid accumulation in epididymal CB1-receptor knockout cells was significantly reduced on days 3 and 6 . In contrast, inguinal CB1-receptor knockout cells showed an increase in differentiation on days 3 and 6 . Furthermore, we analyzed main differentiation markers in these cells at days 3 and 6 after induction. The Preadipocyte factor (Pref 1), which is an inhibitory factor of adipogenesis, was markedly induced with a maximum of nearly $700 \%$ on day 6 in epididymal CB1-receptor knockout cells, whereas in inguinal cells this marker was significantly reduced by $70 \%$. In line with these results, we found a decrease of PPAR $\gamma$, Glut 4 and aP2 in epididymal CB1-receptor knockout fat cells by $40 \%$, $68 \%$ and $52 \%$, respectively. In inguinal CB1-receptor knockout cells, the expression of these late differentiation markers was elevated (233\% PPAR $\gamma, 467 \%$ aP2, 33\% Glut 4) compared with their wild-type controls (Table 1 ).

CB1-receptor knockout enhances insulin sensitivity in inguinal adipocytes

Insulin-induced glucose uptake was found to be impaired in epidiymal CB1-receptor knockout cells by 10\% (1 nM),

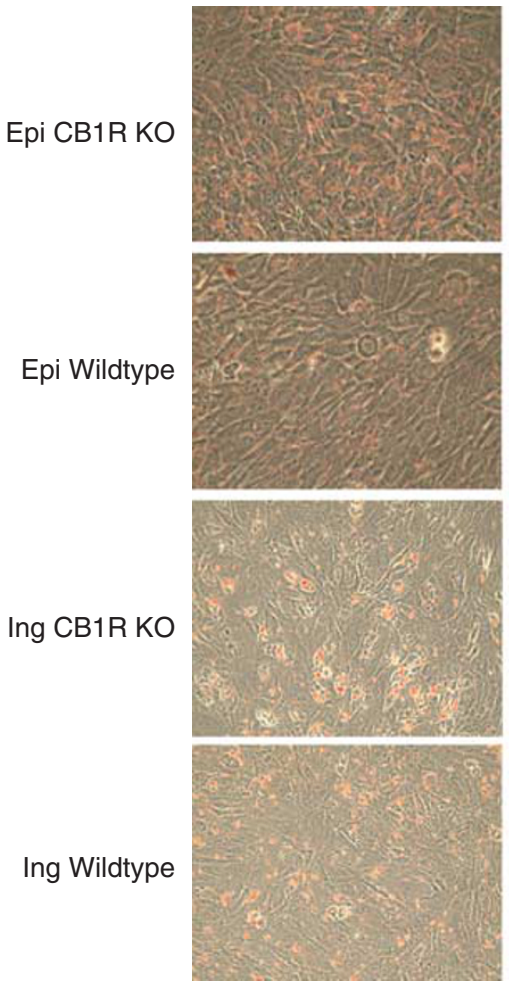

Day 0
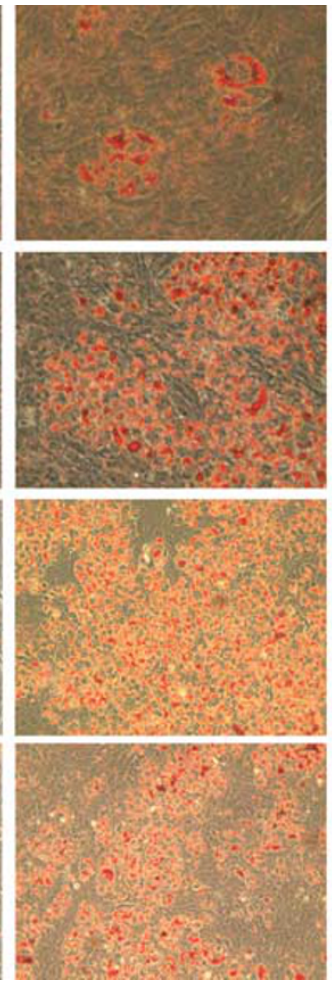

Day 3
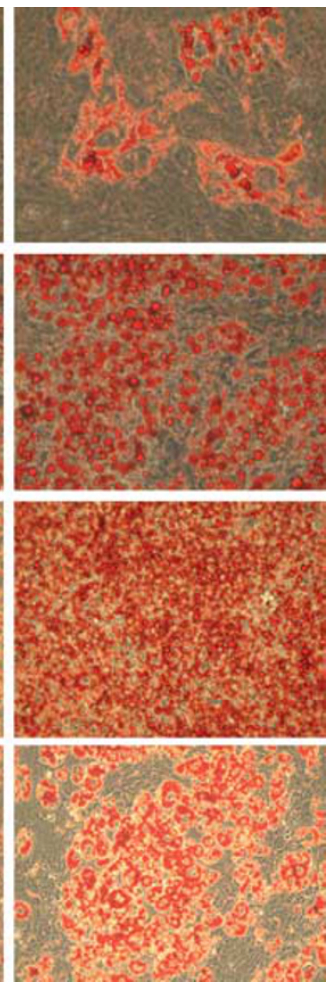

Day 6

Figure 1 Depot-specific effects on adipocyte differentiation are demonstrated. Oil Red O staining of newly generated inguinal (Ing) and epididymal (Epi) CB1receptor knockout (KO) cells compared with their wild-type control. Lipid accumulation was visualized at days 0, 3 and 6 during adipogenesis. Microscopic pictures in 40-fold magnification are shown. 

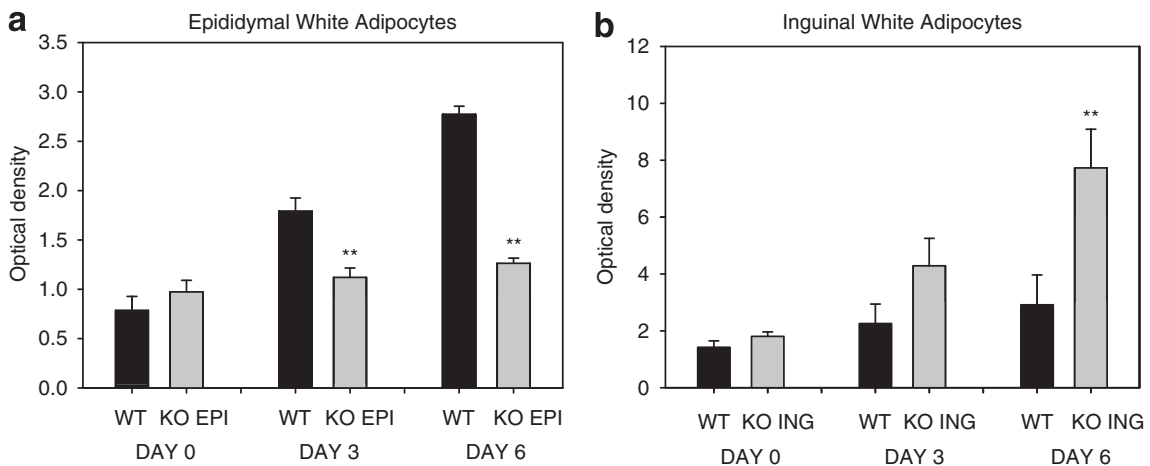

Figure 2 (a, b) Densitometric analysis was performed after Oil Red O staining of the adipocytes. Bar graph analyses showed intensity of Oil Red O (optical density) measured on days 0,3 and 6. CB1-receptor knockout (KO) cells were compared with their wild-type (WT) controls. Eight independent experiments are shown in a and $\mathbf{b}$ as mean \pm s.e.m., ${ }^{*} P<0.05,{ }^{* *} P<0.01$ comparing wild types to CB1-receptor knockout cells. EPI, epididymal; ING, inguinal.

Table 1 Markers of adipocyte differentiation were decreased in epididymal CB1R KO cells, whereas they were enhanced in inguinal CB1R KO adipocytes

\begin{tabular}{|c|c|c|c|c|c|c|c|c|}
\hline \multirow{2}{*}{$\begin{array}{l}\text { Differentiation marker } \\
\text { Day of differentiation }\end{array}$} & \multicolumn{2}{|c|}{ Pref 1} & \multicolumn{2}{|c|}{$P P A R \gamma$} & \multicolumn{2}{|c|}{$a P 2$} & \multicolumn{2}{|c|}{ Glut 4} \\
\hline & Day 3 & Day 6 & Day 3 & Day 6 & Day 3 & Day 6 & Day 3 & Day 6 \\
\hline Epididymal WT vs KO & $\uparrow 121 \%$ & $\uparrow 699 \%$ & $\downarrow 44 \%$ & $\downarrow 39 \%$ & $\downarrow 27 \%$ & $\downarrow 52 \%$ & $\downarrow 35 \%$ & $\downarrow 68 \%$ \\
\hline Inguinal WT vs KO & $\downarrow 69 \%$ & $\downarrow 65 \%$ & $\uparrow 186 \%$ & $\uparrow 233 \%$ & $\uparrow 132 \%$ & $\uparrow 467 \%$ & $\uparrow 49 \%$ & $\uparrow 33 \%$ \\
\hline
\end{tabular}

Abbreviations: CB1R, cannabinoid 1 receptor; KO, knockout; PPAR $\gamma$, peroxisome proliferator-activated receptor $\gamma$; Pref 1, preadipocyte factor; WT, wild type. Table 1a compares epididymal WT cells to epididymal KO cells, whereas in table $1 \mathrm{~b}$ the contrast of inguinal wild-type cells to inguinal KO cells is demonstrated. Data are shown for day 3 and day 6. Pref- 1 is an inhibitory marker of early adipogenesis, whereas aP2, PPAR $\gamma$ and GLUT 4 are favorable markers of late adipogenesis. The percentage of their respective increase $(\uparrow)$ or decrease $(\downarrow)$ is demonstrated in the table. All data are normalized using $36 B 4$ as a housekeeping gene. On average six independent experiments are shown. WT control cells are compared to CB1-receptor knockout cells.

$18 \%(10 \mathrm{~nm})$ and $28 \%(100 \mathrm{nM})$. In contrast, inguinal CB1-receptor knockout cells showed an enhancement in glucose uptake with an increase of $21 \%(1 \mathrm{nM}), 35 \%(10 \mathrm{nM})$, $30 \%$ (100 nM) compared with their wild-type controls (Figure 3$)$.

CB1-receptor knockout induces apoptosis in epididymal but not in inguinal cells

We next assessed the phosphorylation of p53, a marker for apoptosis. We found a highly significant increase of p53 phosphorylation in epididymal knockout cells compared with their control cells. This effect was dose-dependent with a $562 \%$ increase on day 3 and $1329 \%$ on day 6 of the differentiation cycle. In contrast, inguinal CB1-receptor knockout cells showed a reduction in p53 phosphorylation by $57 \%$ on day 3 and $84 \%$ on day 6 (Figure 4 ). To confirm these data, we next examined apoptotic cell death in both cell depots at day 0 . In line with the results for p53 phosphorylation, we also found an increase of cell death in epididymal CB1-receptor knockouts by $\sim 51 \%$, whereas inguinal CB1-receptor knockout cells resulted in a significantly decreased apoptotic rate by $41 \%$ when compared with their control cells (Figure 5).

CB1-receptor knockout influences inflammatory adipokine gene expression in a depot-specific matter

To examine the effect of a CB1-receptor blockade on inflammation, we measured IL-6, monocyte chemoattractant protein 1 (MCP-1) and tumor necrosis factor (TNF)- $\alpha$ mRNA expressions in epididymal and inguinal CB1-receptor knockout preadipocytes (Figure 6). In epididymal knockout cells all proinflammatory markers were elevated. We found a $156 \%$ increase of IL- 6, MCP-1 was induced by $200 \%$ and TNF- $\alpha$ by $192 \%$ compared with epididymal control preadipocytes. In contrast, there was a decrease in inguinal CB1-receptor preadipocytes of IL-6, MCP-1 and TNF- $\alpha$ by $40 \%, 5 \%$ and $23 \%$, respectively. These findings were even more pronounced on day 3 of the differentiation process. An increase in the expression profile of IL-6, MCP-1 and TNF- $\alpha$ by $357 \%, 326 \%$ and $371 \%$, respectively, was found in epididymal CB1-receptor knockout cells, as well as a decreased expression of these inflammation markers in inguinal CB1-receptor knockout cells (IL-6 60\%, MCP-1 26\%, TNF- $\alpha 32 \%$ ) when compared with their wild-type controls.

\section{CB1-receptor knockout enhances brown fat cell marker} expression in inguinal cells, whereas reducing it in epididymal cells

In epididymal cells, the expression of PGC- $1 \alpha$, a transcriptional activator of PPAR $\gamma$ on the UCP-1 promoter, was slightly but not significant reduced by $23 \%$ in preadipocytes (because of high standard deviation results were not significant), but was significantly decreased by $64 \%$ in mature cells when compared with controls. In contrast, 
a Epididymal White Adipocytes

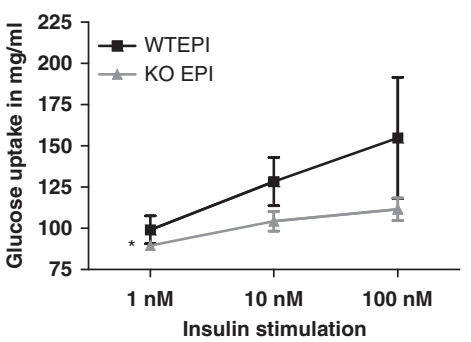

\begin{tabular}{|c|c|c|c|}
\hline Insulinstimulation & 1nM Insulin & 10nM Insulin & 100nM Insulin \\
\hline \hline WT EPI & 99.1 & 128.35 & 154.85 \\
\hline KO EPI & $89.6^{*}$ & 104.4 & 111.53 \\
\hline
\end{tabular}

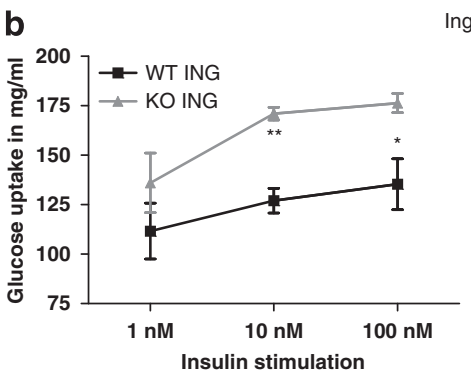

\begin{tabular}{l|r}
\hline 111.67 & 127.0 \\
\hline 136.0 & $171.0^{*}$
\end{tabular}

\begin{tabular}{l|c|c|c} 
KO ING & 136.0 & $171.0^{\star *}$ & 176.53 \\
\end{tabular}

Figure 3 (a, b) CB1-receptor knockout $(\mathrm{KO})$ influences insulin sensitivity in a depot-specific manner. Glucose uptake was measured using 2-desoxy-3H glucose. Mature fat cells were stimulated with different insulin concentrations ( $1 \mathrm{nM}, 10 \mathrm{nM}$ and $100 \mathrm{nM}$ insulin per well) for 30 min. The black line shows the wild-type (WT) control, whereas the gray line illustrates the KO adipocytes from inguinal (ING) and epididymal (EPI) fat depots as indicated. Four independent experiments are shown as mean \pm s.e.m., ${ }^{*} P<0.05,{ }^{* *} P<0.01$ comparing wild types to CB1-receptor $\mathrm{KO}$ cells.

a
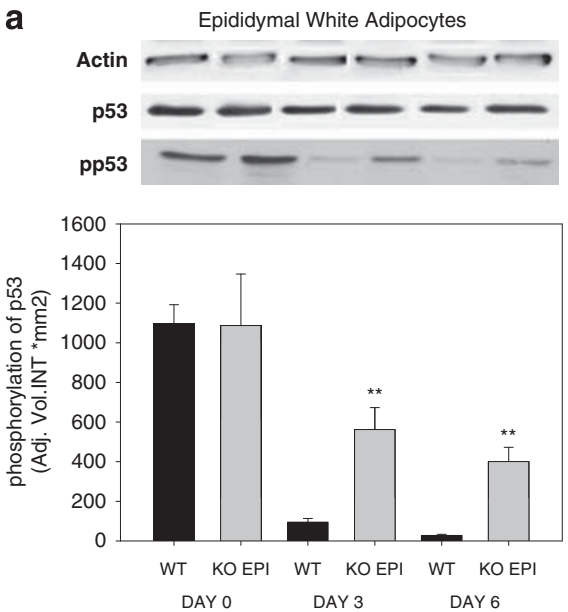

b
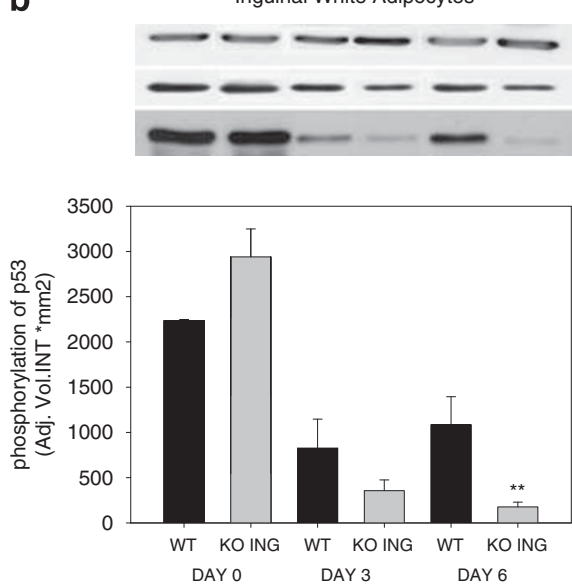

Figure 4 (a, b) CB1-receptor knockout (KO) enhances p53 phosphorylation in epididymal (EPI) cells while reducing it in inguinal (ING) adipocytes. (a) The comparison between wild-type controls (black bars) and phopho-p53 expression in epididymal KO cells (gray bars), whereas b highlights the differences of phosphop53 expression levels of wild-type (WT) control cells and inguinal KO cells. Bar graph analysis of four independent experiments are shown as mean $\pm \mathrm{s}$.e.m. ${ }^{*} P<0.05,{ }^{* *} P<0.01$ comparing WT control cells to CB1-receptor KO cells. A representative western blot image is inserted.

inguinal preadipocytes showed a highly significant increase of PGC- $1 \alpha$ expression by $152 \%$ in preadipocytes and a significant enhancement of $315 \%$ in mature cells (Figure 7 ). In line with these results, the expression of UCP-1 did not differ significantly in epididymal preadipocytes, whereas the mature cells showed a significantly decreased expression by $57 \%$ of this brown fat cell marker. There was an increased basal expression of UCP-1 in inguinal CB1-receptor knockout cells by $252 \%$ at day 0 and $243 \%$ at day 6 compared with inguinal control cells (Figure 8).

\section{CB1-receptor knockout promotes mitochondrial biogenesis} in inguinal white adipocytes

In consequence of our findings of an increased PGC- $1 \alpha$ and UCP-1 content in inguinal CB1-receptor knockout 


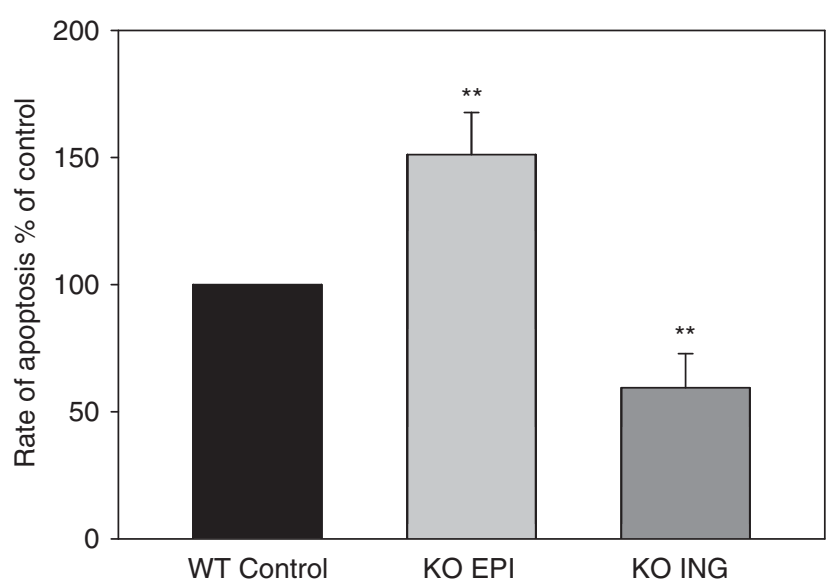

Figure 5 Apoptosis is induced in epidiymal (EPI) CB1-receptor knockout (KO) cells. A photometric enzyme immunoassay for the determination of cytoplasmic histone-associated DNA fragments after cell death was performed. Data are shown at day 0. Bar graph analysis of 10 independent experiments are shown as mean \pm s.e.m., ${ }^{*} P<0.01$ comparing wild-type (WT) control cells to CB1-receptor KO cells. ING, inguinal. adipocytes, we investigated the expression of mitochondrial markers during adipogenesis in these cells compared with epididymal CB1-receptor knockout cells. Although there were no changes in Tfam and NRF-1 expression in preadipocytes, we found an increase of both markers in inguinal adipocytes on days 3 and 6 of the differentiation process. In epididymal cells, the expression of these markers was unaltered (data not shown). To confirm an induction of mitochondrial biogenesis, we further used a mitochondrialspecific fluorescent staining. We found a significant decrease of fluorescent intensity in epididymal cells by $20 \%$. In contrast, the fluorescent intensity in inguinal cells was increased by $52 \%$ (Figure 9 ).

\section{Oxygen consumption is increased in inguinal CB1-receptor knockout cells}

We finally examined the rate of oxygen consumption as a cellular read-out for increased respiratory chain activity. Epididymal CB1-receptor knockout cells displayed a reduced rate of oxygen consumption by $30 \%$ at day 0 and $27 \%$ at
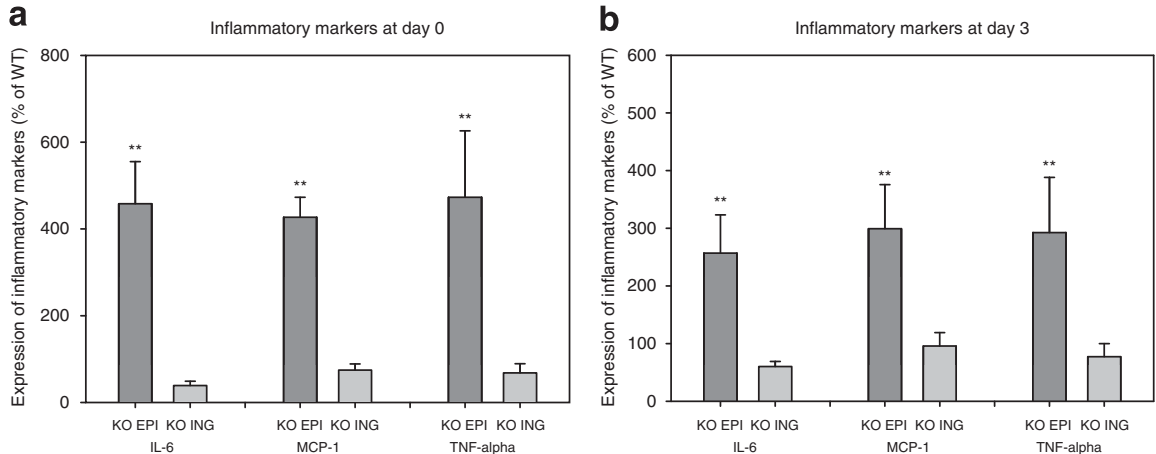

Figure $6(\mathbf{a}, \mathbf{b})$ Expression of inflammation markers was elevated in epididymal (EPI) knockout (KO) cells and decreased in inguinal (ING) KO cells, which was quantified using real-time PCR. Data of inflammation markers including IL-6, monocyte chemoattractant protein 1 (MCP- 1 ) and TNF- $\alpha$ are shown in $\mathbf{a}$ at day 0 and $\mathbf{b}$ at day 3. The dark gray bars show the increase of expression levels of EPI KO cells in $\%$ and the light gray bars demonstrate the reduction of inflammation markers in ING KO cells in \% compared with their individual wild-type controls (set as 100\%). All data were normalized using 36B4 as a housekeeping gene. Of each inflammation marker six independent experiments are shown as mean \pm s.e.m., ${ }^{*} P<0.05,{ }^{* *} P<0.01$ comparing wild-type control cells to CB1-receptor KO cells.
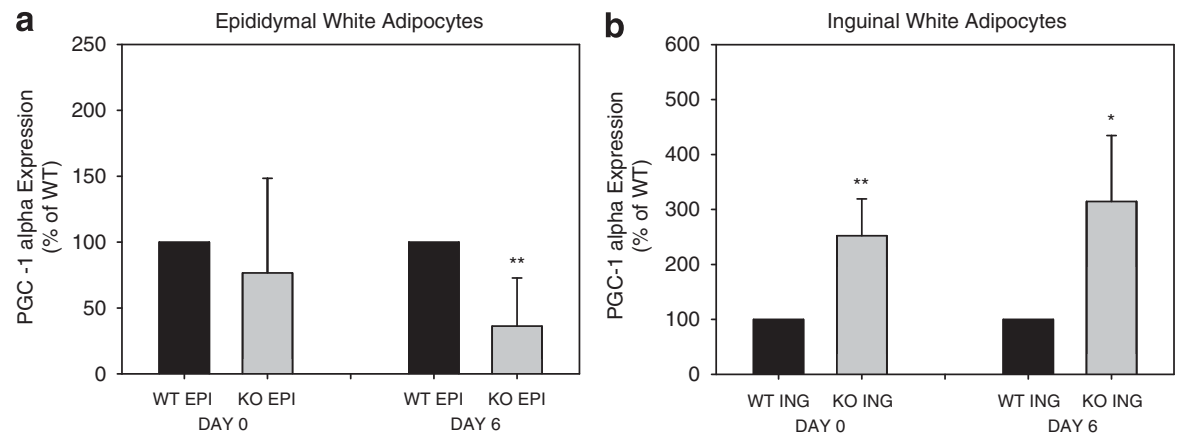

Figure $7(\mathbf{a}, \mathbf{b})$ PGC-1 $\alpha$ expression is increased in inguinal (ING) knockout (KO) cells. Expression is illustrated at days 0 and 6 during the differentiation process. (a) Compares PGC-1 $\alpha$ expression of epididymal wild-type (WT) cells and epididymal (EPI) CB1-receptor KO cells, (b) shows PGC-1 $\alpha$ expression of ING WT cells compared with ING CB1-receptor KO cells. All data normalized using 36B4 as a housekeeping gene. Six independent experiments are shown as mean \pm s.e.m., ${ }^{*} P<0.05,{ }^{* *} P<0.01$ comparing WT control cells to CB1-receptor KO cells. 

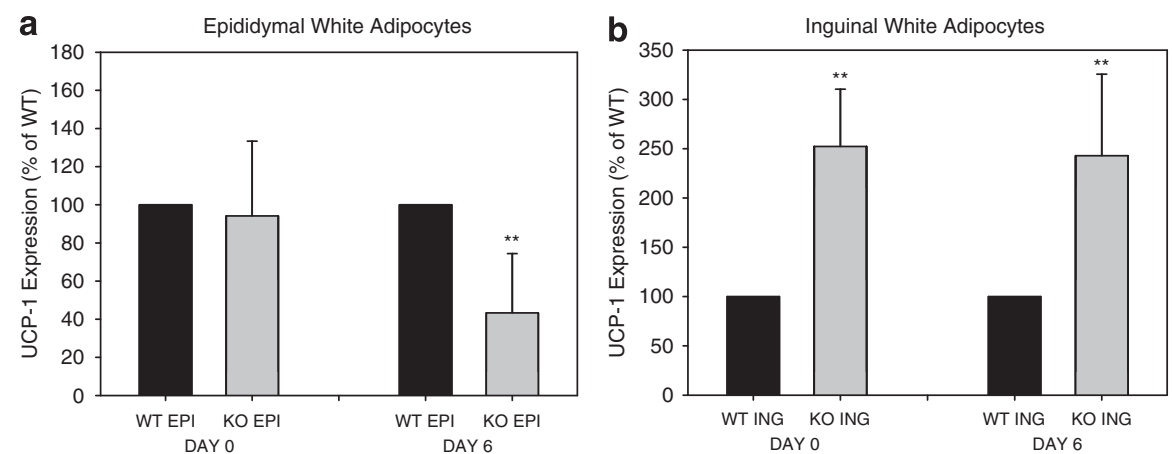

Figure 8 (a, b) The expression level of UCP-1 (uncoupling protein-1) mRNA was enhanced in inguinal (ING) knockout (KO) cells at day 0 and at day 6 . In a, the black bars represent epididymal (EPI) wild-type (WT) controls and the gray bars show EPI KO cells. (b) Illustrates the UCP-1 expression level of ING KO cells compared with their WT control cells. All data were normalized using 36B4 as a housekeeping gene. Six independent experiments are shown as mean \pm s.e.m., ${ }^{\star} P<0.05$, ${ }^{* *} P<0.01$ comparing WT control cells to CB1-receptor KO cells.

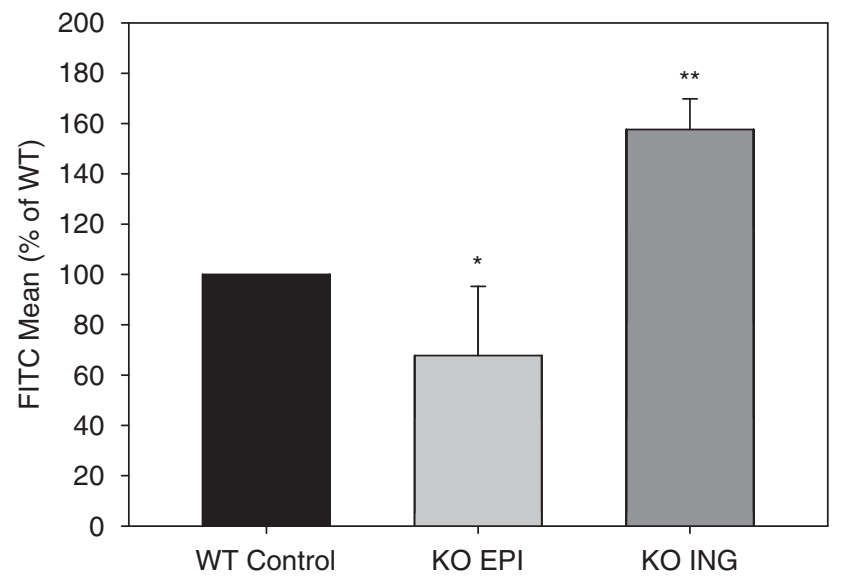

Figure 9 The amount of mitochondria was increased in inguinal (ING) knockout (KO) cells. After Mitotracker Green staining, fluorescent intensity was determined by FACS and analyzed by the Flow Software. Mitochondrial biogenesis was measured and compared with wild-type (WT) control cells (100\%). A decreased amount of labelled mitochondria of epididymal (EPI) KO cells and an increase of the amount of mitochondria of ING KO cells was observed. A number of six independent experiments are shown as mean \pm s.e.m., ${ }^{*} P<0.05,{ }^{*} P<0.01$ comparing WT control cells to CB1-receptor $\mathrm{KO}$ cells.

day 6 compared with wild-type controls. In contrast, oxygen consumption in inguinal CB1-receptor knockout adipocytes was found to be significantly elevated by $83 \%$ at day 0 and by $92 \%$ at day 6 compared with wild-type controls (Figure 10).

\section{Discussion}

To distinguish between different fat depot functions has become the focus of intensive research recently. Visceral fat distribution in obesity is strongly linked to metabolic complications of obesity because of a greater infiltration of adipose tissue with inflammatory cells, excess release of potentially harmful cytokines and reduced release of beneficial adipokines. ${ }^{11,30}$ In contrast, abdominal subcutaneous fat is not associated with a linear increase in the prevalence for metabolic and cardiovascular diseases. Our results show, for the first time, a depot-specific effect of a CB1-receptor knockout in adipocytes. Interestingly, CB1-receptor knockout cells promote a metabolically beneficial status for subcutaneous fat cells, such as increased insulin sensitivity, decreased inflammation and an enhancement in mitochondrial biogenesis combined with an induction of oxygen consumption when compared with epididymal wild-type cells. The EC system has a major role in controlling energy homeostasis via central and peripheral pathways. Clinical trials showed that blocking of the CB1-receptor, with the selective antagonist rimonabant, leads to an induction of weight loss and improves cardiovascular risk factors in obese patients. ${ }^{7,31-33}$ CB1-receptor knockout mice are protected against diet-induced obesity. ${ }^{6,34}$ Cell proliferation and lipid accumulation are the major factors contributing to fat mass development in obesity. Our study demonstrates a depotspecific effect on adipocyte differentiation. In newly generated CB1-receptor knockout cell lines from different fat cell depots, we found an impaired differentiation together with a decreased expression pattern of differentiation markers in epididymal cells compared with their wild-type control cells. Inguinal cells, on the other hand, showed an accelerated accumulation of lipids accompanied with an increase of differentiation markers. Effects of ECs on adipogenesis were investigated in 3T3-L1 adipocytes. Blocking the CB1-receptor arrested adipocyte proliferation. ${ }^{35}$ Furthermore, increased EC levels promote preadipocyte differentiation and chronic stimulation of the CB1-receptor during adipogenesis induces the accumulation of lipids and the elevation of PPARã as a differentiation marker. ${ }^{36,37}$ These results are in line with our findings for epididymal fat cells with a lack of differentiation after CB1-receptor blockage. Thus, blocking the CB1-receptor in adipocytes has an important impact on fat composition by lipid deposition in inguinal fat on the one hand 

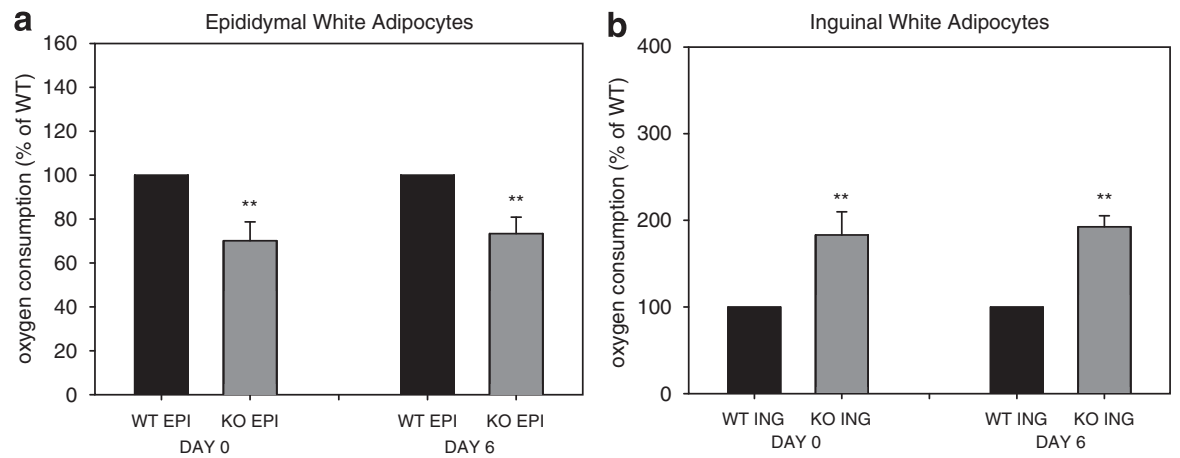

Figure $10(\mathbf{a}, \mathbf{b})$ Oxygen consumption was elevated in inguinal (ING) knockout (KO) cells. The oxygen consumption was detected at day 0 and at day 6 when the cells were fully differentiated. In a, the results of epididymal (EPI) wild-type (WT) cells compared with EPI KO cells are shown. Whereas in $\mathbf{b}$ the ING WT cells are demonstrated in contrast to inguinal KO cells. Here 12 independent experiments are shown as mean \pm s.e.m., ${ }^{*} P<0.05,{ }^{*} P<0.01$ comparing WT control cells to CB1-receptor KO cells.

while reducing epididymal fat mass on the other. Higher amounts of lower subcutaneous body fat in humans seem to be associated with a reduced risk of metabolic complications. ${ }^{38}$ Similar observations are shown for selective PPAR $\gamma$ agonists. Thiazolidinediones may induce differentiation of human preadipocytes isolated from subcutaneous fat but not those from visceral fat. ${ }^{39,40}$ Treatment with PPAR $\gamma$ agonists in rats also redistributes fat by stimulating the lipid uptake and esterification potential in subcutaneous fat and by augmentation of oxygen consumption. Conversely, lipid uptake is minimally altered and energy expenditure is greatly increased in visceral fat, with consequent reduction in fat accumulation. $^{41}$

Recent studies describe a depot-specific effect on inguinal and epididymal differentiation in cells treated with dehydroepiandrosterone. Again, inguinal preadipocyte differentiation was unaffected or increased, whereas omental preadipocytes showed significantly reduced adipogenesis. ${ }^{42}$

Reduced adipogenesis might be explained by the induction of apoptosis. Hallenborg et al. ${ }^{43}$ showed that p53 inhibits adipogenesis and maintains adipose tissue function. We demonstrate a significant increase in p53 phosphrylation in mature epididymal cells compared with their wild-type controls. In contrast, p53 phosphorylation in inguinal adipocytes was impaired. These data are in line with other findings, showing that omental preadipocytes are more susceptible to apoptotic stimuli than subcutaneous preadipocytes. ${ }^{44}$ There are also a lot of other stimuli that have shown to influence the differentiation process and apoptotic rate in adipose tissue. For example, after Ajoene (a garlic extract), Guggulsterone, Withaferin A, Chlorella methanol and Resveratrol (ingredient of red wine) stimulation or treatment, a reduced lipid accumulation and adipogenesis and an increase in the number of apoptotic cells was noted. ${ }^{4-48}$ Altering fat mass by directly affecting cell viability, adipogenesis and apoptosis, may have applications for the treatment of obesity and might be one mechanism for the beneficial effect of blocking the CB1-receptor with rimonabant. In clinical trials with rimonabant, patients mainly loose visceral fat, which is evidenced by a reduced waist to hip circumference. Redistributing fat from lipolytic visceral fat to more anabolic subcutaneous fat is thought to have a role in the insulin-sensitizing effect of PPAR $\gamma$ agonists in humans. ${ }^{41}$ These results are consistent with our data showing an elevated glucose uptake in inguinal CB1-receptor knockout adipocytes and again underline the insulin-sensitizing effect of rimonabant in obese patients.

Obesity is associated with chronic inflammation. During the development of obesity macrophages infiltrate adipose tissue. This seems to be more pronounced in visceral adipose tissue rather than in subcutaneous fat. ${ }^{49}$ In extremely obese patients, the visceral fat is the main contributor to high plasma IL- 6 concentrations. ${ }^{50}$ Moreover, MCP- 1 and TNF- $\alpha$ are related to adipocyte dedifferentiation and systematic insulin resistance. ${ }^{51,52}$ As compared with subcutaneous adipose tissue, visceral adipose tissue endocrine activity is considered to be predominant in contributing to chronic low-grade inflammation found in obese subjects. This is in line with our findings demonstrating that CB1-receptor knockout cells mediate a proinflammatory status by enhancing IL-6, MCP-1 and TNF- $\alpha$ expression in epididymal cells, whereas reducing these inflammation markers in inguinal cells.

The conversion of white adipocytes into a thermogenic active brown fat phenotype is a promising strategy to control energy homeostasis. We and others have recently published direct effects of CB1-receptor blockade on thermogenesis, mitochondrial biogenesis and oxidative metabolism. A pharmaceutical blockade of the receptor with rimonabant results in a transdifferentiation of white cells into a brown fat cell phenotype. ${ }^{3,8}$ Both studies did not distinguish between white fat depots. Only Jourdan et al. ${ }^{53}$ examined the impact of CB1-receptor antagonism on liver and adipose tissue metabolism in a mouse model of diet-induced obesity. His research group demonstrated that in visceral but not 
subcutaneous fat, genes that are involved in transport, synthesis, oxidation, and release of fatty acidy were upregulated by HSHF feeding, whereas this effect was counteracted by CB1-receptor antagonism. ${ }^{53}$ They evaluated the determining factor for the reversion in liver steatosis that can be induced by rimonabant (SR141716) and found, in line with our data, that the blockage of the EC system can have different effects on visceral and subcutaneous fat. TNF- $\alpha$ expression was reduced in subcutaneous adipose tissue after treatment with a CB1-receptor antagonist but inflammation remained high in visceral adipose tissue, as confirmed in our experiments. Furthermore, Jourdan et al. ${ }^{53}$ showed an increased expression of PPAR $\gamma$ in subcutaneous adipose tissue after CB1-receptor blockage. In addition, we were able to demonstrate that there is not only a differentiation benefit in the subcutaneous depot but also furthermore a lack of differentiation in the metabolically harmful visceral depot. Furthermore, we observed, in inguinal CB1-receptor knockout cells, a depot-specific effect of transdifferentiation in white adipocytes. UCP- 1 as well as PGC- $1 \alpha$ expressions were significantly increased in inguinal CB1-receptor knockout cells, whereas they were downregulated or not affected in epididymal cells. Moreover, mitochondrial biogenesis in inguinal CB1-receptor knockout cells were elevated together with an increase in oxygen consumption as a final cellular read-out for increased activity of the respiratory chain. In contrast, epididymal CB1-receptor knockout cells showed impairment in both, mitochondrial biogenesis and oxidative metabolism. Thus, inguinal cells appear to be more sensitive toward transdifferentiation. The higher expression levels of markers for brown adipocytes and an increased mitochondrial biogenesis in cells that were isolated from subcutaneous tissues of CB1-receptor knockout mice might partly be due to the differentiation benefit we noted in these cells compared with their wild-type controls. In addition, there is likely to exist another process that induces the transdifferentiation as we already noted differences at day 0 during the differentiation process independent of lipid accumulation. Relevant signaling mechanisms currently remain unclear. Interestingly, new studies show that PRDM 16, a key factor for brown fat cell development, is five- to six-fold higher expressed in inguinal cells than in epididymal adipocytes, suggesting that these cells have more potential to convert into brown fat cells. Previous studies have demonstrated that UCP-1 expression in white adipocytes is increased after chronic $\beta$-adrenergic stimulation or stimulation with a PPAR $\gamma$ agonist. Again this phenomenon is particularly evident in inguinal depots and less in epididymal adipocytes. ${ }^{54,55}$ Moreover, rosiglitazone promotes mitochondrial biogenesis in vivo in white fat of ob/ob mice as well as in human subcutaneous fat. ${ }^{56}$ However, emergence of brown fat cells in white fat depots is associated with a lean phenotype in transgenic mouse models (FOXC2, 4E-BP1). ${ }^{24,25}$ These mice have an enhanced metabolic rate and insulin sensitivity and are protected against diet-induced obesity. Furthermore, transgenic mice expressing UCP-1 in white adipose tissue are protected against genetic and dietary obesity and show an increase in white adipose tissue oxygen consumption. ${ }^{57}$

Developing strategies to enhance brown fat cell cluster in white adipose tissue depots by converting white adipocytes might be a tool to treat obesity and associated disorders.

In summary, the present study reveals a depot-specific effect of cannabinoid action in adipose cells from different fat depots. In newly generated CB1-receptor knockout adipose cell lines, this study demonstrates a deficit in differentiation and an increase in apoptosis in epididymal fat cells accompanied by increased inflammation and reduced oxygen consumption. In contrast, inguinal CB1-receptor knockout cells show less inflammation and tend to be more sensitive toward a conversion into a thermogenic active brown fat phenotype. These findings elucidate peripheral mechanisms resulting in beneficial effects on energy balance achieved by blockade of the EC system. Enhanced thermogenesis represents a peripheral mechanism contributing to weight loss and improving glucose homeostasis in patients treated with a CB1-receptor antagonist. In addition, CB1receptor blockade promotes the development of subcutaneous fat and reduces visceral fat, which is associated with metabolic complications of obesity. To use this new understanding of CB1-receptor blockade for the benefit in obesity treatment the development of adipocyte-specific CB1-receptor antagonists should be intensified.

\section{Conflict of interest}

The authors declare no conflict of interest.

\section{Acknowledgements}

The CB1-receptor knockout mice were kindly provided by Sanofi-Aventis. This study was supported by a translational research grant from Sanofi-Aventis, Germany, and a grant from the Deutsche Forschungsgemeinschaft to JK: (KL 1131/4-1).

\section{References}

1 Tiraby C, Langin D. Conversion from white to brown adipocytes: a strategy for the control of fat mass? Trends Endocrinol Metab 2003; 14: 439-441.

2 Tiraby C, Tavernier G, Lefort C, Larrouy D, Bouillaud F, Ricquier $\mathrm{D}$ et al. Acquirement of brown fat cell features by human white adipocytes. J Biol Chem 2003; 278: 33370-33376.

3 Perwitz N, Wenzel J, Wagner I, Büning J, Drenckhan M, Zarse K et al. Cannabinoid type 1 receptor blockade induces towards a brown fat phenotype in white adipocytes. Diabetes Obes Metab 2010; 12: 158-166.

4 Pagotto U, Vicennati V, Pasquali R. The endocannabinoid system and the treatment of obesity. Ann Med 2005; 37: 270-275.

5 Perwitz N, Fasshauer M, Klein J. Cannabinoid receptor signaling directly inhibits thermogenesis and alters expression of adiponectin and visfatin. Horm Metab Res 2006; 38: 356-358. 
6 Ravinet Trillou C, Delgorge C, Menet C, Arnone M, Soubrie P. CB1 cannabinoid receptor knockout in mice leads to leanness, resistance to diet-induced obesity and enhanced leptin sensitivity. Int J Obes Relat Metab Disord 2004; 28: 640-648.

7 Van Gaal LF, Rissanen AM, Scheen AJ, Ziegler O, Rossner S. Effects of the cannabinoid-1 receptor blocker rimonabant on weight reduction and cardiovascular risk factors in overweight patients: 1-year experience from the RIO-Europe study. Lancet 2005; 365: 1389-1397.

8 Tedesco L, Valerio A, Cervino C, Cardile A, Pagano C, Vettor R et al. Cannabinoid type 1 receptor blockade promotes mitochondrial biogenesis through endothelial nitric oxide synthase expression in white adipocytes. Diabetes 2008; 57: 2028-2036.

9 Hajer GR, van Haeften TW, Visseren FL. Adipose tissue dysfunction in obesity, diabetes, and vascular diseases. Eur Heart J 2008; 29: 2959-2971.

10 Porter SA, Massaro JM, Hoffmann U, Vasan RS, O'Donnel CJ, Fox CS. Abdominal subcutaneous adipose tissue: a protective fat depot? Diabetes Care 2009; 32: 1068-1075.

11 Jensen MD. Role of body fat distribution and the metabolic complications of obesity. J Clin Endocrinol Metab 2008; 93: S57-S63.

12 Ibrahim M. Subcutaneous and visceral adipose tissue:structural and functional differences. Obes Rev 2010; 1: 11-18.

13 Klein JPPA, Owecki M, Chaldokov GN, Böhm M, Hausman G, Lapiere CM et al. What are subcutaneous adipocytes really good for? Exp Dermatol 2007; 1: 45-70.

14 Tran TT, Kahn CR. Transplantation of adipose tissue and stem cells:role in metabolism and disease. Nat Rev Endocrinol 2010; 4: 195-213.

15 Tran TT, Yamamoto Y, Gesta S, Kahn CR. Beneficial effects of subcutaneous fat transplantation on metabolism. Cell Metab 2008; 7: 410-420.

16 Tritos NA, Mantzoros CS. Leptin: its role in obesity and beyond. Diabetologia 1997; 40: 1371-1379.

17 Yang $\mathrm{X}$, Smith U. Adipose tissue distribution and risk of metabolic disease: does thiazolidinedione-induced adipose tissue redistribution provide a clue to the answer? Diabetologia 2007; 50: 1127-1139.

18 Ravussin E, Galgani JE. The implication of brown adipose tissue for humans. Annu Rev Nutr 2011; 31: 33-47.

19 Richard DCA, Dore G, Ouellet V, Picard F. Determinants of brown adipocyte development and thermogenesis. Int J Obes 2010; 34: S59-S66.

20 Richard DPF. Brown fat biology and thermogenesis. Front Biosci 2011; 16: 1233-1260.

21 Tews DWM. Renaissance of Brown Adipose Tissue. Horm Res Paediatr 2011; 75: 231-239.

22 Puigserver P, Wu Z, Park CW, Graves R, Wright M, Spiegelman BM. A cold-inducible coactivator of nuclear receptors linked to adaptive thermogenesis. Cell 1998; 92: 829-839.

23 Medina-Gomez G, Gray S, Vidal-Puig A. Adipogenesis and lipotoxicity: role of peroxisome proliferator-activated receptor gamma (PPARgamma) and PPARgammacoactivator-1 (PGC1). Public Health Nutr 2007; 10: 1132-1137.

24 Tsukiyama-Kohara K, Poulin F, Kohara M, DeMaria CT, Cheng A, $\mathrm{Wu} \mathrm{Z}$ et al. Adipose tissue reduction in mice lacking the translational inhibitor 4E-BP1. Nat Med 2001; 7: 1128-1132.

25 Cederberg A, Gronning LM, Ahren B, Tasken K, Carlsson P, Enerback S. FOXC2 is a winged helix gene that counteracts obesity, hypertriglyceridemia, and diet-induced insulin resistance. Cell 2001; 106: 563-573.

26 Seale PKS, Spiegelman B. Transcriptional control of brown adipocyte development and physiological function-of mice and men. Genes Dev 2009; 23: 788-797.

27 Klein J, Fasshauer M, Ito M, Lowell BB, Benito M, Kahn CR. Beta(3)-adrenergic stimulation differentially inhibits insulin signaling and decreases insulin-induced glucose uptake in brown adipocytes. J Biol Chem 1999; 274: 34795-34802.
28 Klein J, Fasshauer M, Klein $\mathrm{HH}$, Benito M, Kahn CR. Novel adipocyte lines from brown fat: a model system for the study of differentiation, energy metabolism, and insulin action. Bioessays 2002; 24: 382-388.

29 Pfaffl MW, Horgan GW, Dempfle L. Relative expression software tool (REST) for group-wise comparison and statistical analysis of relative expression results in real-time PCR. Nucleic Acids Res 2002; 30: e36.

30 Jensen MD. Role of body fat distribution and the metabolic complications of obesity. J Clin Endocrinol Metab 2008; 93: 57-63.

31 Despres JP, Golay A, Sjostrom L. Effects of rimonabant on metabolic risk factors in overweight patients with dyslipidemia. N Engl J Med 2005; 353: 2121-2134.

32 Pi-Sunyer FX. The relation of adipose tissue to cardiometabolic risk. Clin Cornerstone 2006; 8 (Suppl 4): S14-S23.

33 Scheen AJ. Does the metabolic syndrome help to select patients requiring high statin dose? Lancet 2006; 368: 893-894.

34 Cota D, Marsicano G, Tschop M, Grubler Y, Flachskamm C, Schubert $\mathrm{M}$ et al. The endogenous cannabinoid system affects energy balance via central orexigenic drive and peripheral lipogenesis. J Clin Invest 2003; 112: 423-431.

35 Gary-Bobo M, Elachouri G, Scatton B, Le Flur G, Oury-Donat F, Bensaid M. The cannabinoid CB1 receptor antagonist rimonabant (SR 141716) inhibits cell proliferation and increases markers of adipocyte maturation in cultured mouse 3T3 F 442A preadipocytes. Mol Pharmacol 2006; 69: 471-478.

36 Matias I, Di Marzo V. Endocannabinoids and the control of energy balance. Trends Endocrinol Metab 2007; 18: 27-37.

37 Matias IG, Gontheir MP, Orlando P, Martiadis V, De Petrocellis L, Cervico $\mathrm{C}$ et al. Regulation, function and dysregulation of endocannabinoids in models of adipose and beta-pencreatic cells and in obesity and hyperglycemia. J Clini Endocrinol Metab 2006; 91: 3171-3180.

38 Snijder MB, Dekker JM, Visser M, Bouter LM, Stehouwer CD, Yudkin JS et al. Trunk fat and leg fat have independent and opposite associations with fasting and postload glucose levels: the Hoorn study. Diabetes Care 2004; 27: 372-377.

39 Adams M, Montague CT, Prins JB, Holder JC, Smith SA, Sanders L et al. Activators of peroxisome proliferator-activated receptor gamma have depot-specific effects on human preadipocyte differentiation. J Clin Invest 1997; 100: 3149-3153.

40 Sewter CP, Blows F, Vidal-Puig A, O’Rahilly S. Regional differences in the response of human pre-adipocytes to PPARgamma and RXRalpha agonists. Diabetes 2002; 51: 718-723.

41 Laplante M, Festuccia WT, Soucy G, Gelinas Y, Lalonde J, Berger JP et al. Mechanisms of the depot specificity of peroxisome proliferator-activated receptor gamma action on adipose tissue metabolism. Diabetes 2006; 55: 2771-2778.

42 Rice SP, Zhang L, Grennan-Jones F, Agarwal N, Lewis MD, Rees DA, Ludgate M. Dehydroepandrosterone (DHEA) treatment in vitro inhibits adipogenesis in human omental but not subcutaneous adipose tissue. Mol Cell Endocrinol 2010; 320: 51-57.

43 Hallenborg PFS, Madsen L, Kristiansen K. The tumor suppressors $\mathrm{pRB}$ and p 53 as regulators of adipocyte differentiation and function. Expert Opin Ther Targets 2009; 13: 235-246.

44 Niesler CU SK, Prins JB. Human preadipocytes display a depotspecific susceptibility to apoptosis. Diabetes 1998; 47: 1365-1368.

45 Ambati S, Yang JY, Rayalam S, Park HJ, Della-Ferra MA, Baile CA. Ajoene exerts potent effects in 3T3-L1 adipocytes by inhibiting adipogenesis and inducing apoptosis. Phytother Res 2009; 23: 513-518.

46 Yang JY, D -FM, Baile CA. Guggulsterone inhibits adipocyte differentiation and induces apoptosis in 3T3-L 1 cells. Obesity (Silver Spring) 2008; 16: 16-22.

47 Park HJ, RS, Della-Ferra MA, Ambati S, Yang JY, Baile CA. Withaferin A induces apoptosis and inhibits adipogenesis in 3T3-L1 adipocytes. Biofactors 2008; 33: 137-148.

48 Chon JW, SJ, Hwang EJ, Park YK. Chlorella methanol extract reduces lipid accumulation in and increases the number of apoptotic 3T3-L1 cells. Ann NY Acad Sci 2009; 1171: 183-189. 
49 Cancello R, Tordjman J, Poitou C, Guilhem G, Bouillot JL, Hugol D et al. Increased infiltration of macrophages in omental adipose tissue is associated with marked hepatic lesions in morbid human obesity. Diabetes 2006; 55: 1554-1561.

50 Fontana L, EJ, Trujillo ME, Scherer PE, Klein S. Visceral fat adipokine secretion is associated with systemic inflammation in obese humans. Diabetes 2007; 56: 1010-1013.

51 Bose T, Alvarenga JC, Tejero ME, Voruganti VS, Proffitt JM, Freeland-Graves JH, Cole SA et al. Association of monocyte chemoattractant protein-1 with adipocyte number, insulin resistance and liver function markers. J Med Primatol 2009; 38: 418-424.

52 Van den Oever IA, RHG, Numohamed MT, Simsek S. Endothelial dysfunction, inflammation and apoptosis in diabetes mellitus. Mediators Inflamm 2010; 2010: 792393.

53 Jourdan T, Djaouti L, Demizieux L, Gresti J, Verges B, Degrace P. CB1 antagonism exerts specific molecular effects on visceral and subcutaneous fat and reverses liver steatosis in diet-induced obese mice. Diabetes 2010; 59: 926-934.

54 Fukui Y, Masui S, Osada S, Umesono K, Motojima K. A new thiazolidinedione, NC-2100, which is a weak PPAR-gamma activator, exhibits potent antidiabetic effects and induces uncoupling protein 1 in white adipose tissue of KKAy obese mice. Diabetes 2000; 49: 759-767.

55 Xue Y, Zhou Y, Wu T, Zhu T, Ji X, Kwon YS et al. Genome-wide analysis of PTB-RNA interactions reveals a strategy used by the general splicing repressor to modulate exon inclusion or skipping. Mol Cell 2009; 36: 996-1006.

56 Wilson-Fritch L, Burkart A, Bell G, Mendelson K, Leszyk J, Nicoloro $\mathrm{S}$ et al. Mitochondrial biogenesis and remodeling during adipogenesis and in response to the insulin sensitizer rosiglitazone. Mol Cell Biol 2003; 23: 1085-1094.

57 Stefl B, Janovska A, Hodny Z, Rossmeisl M, Horakova M, Syrovy I et al. Brown fat is essential for cold-induced thermogenesis but not for obesity resistance in aP2-Ucp mice. Am J Physiol 1998; 274: E527-E533.

This work is licensed under the Creative Commons Attribution-NonCommercial-No Derivative Works 3.0 Unported License. To view a copy of this license, visit http://creativecommons.org/ licenses/by-nc-nd/3.0/ 